\title{
World Cancer Day 2021: Prevention, awareness and accountability in tobacco control
}

Yannick Romero'

Today is World Cancer Day. For 21 years, this day has been synonymous with solidarity, compassion and public awareness.

The year 2020 was unique, not only for the cancer community but for everyone globally as we have all faced the challenge of COVID-19. In a year full of uncertainty, the work done by millions of people in healthcare and research worldwide was brought into the public domain. We also witnessed how fragile health systems are and how cancer care has experienced serious disruptions: early detection and treatment therapies were postponed and the overall care of people living with cancer was delayed, in particular because of fears of getting sick, as cancer is one of the comorbidities that can lead to more serious cases of COVID-19.

On 4 February 2021, we celebrate the 21 st anniversary of World Cancer Day $^{1}$. This day has always been the occasion to offer a message of hope for cancer patients and survivors, particularly this year, thanks to the development of vaccines and the relief they will bring to health systems and those working on the frontlines. The pandemic period is not over, and we will pay the consequences for the next years, but we have now turned a corner and we now know that governments must invest in health systems, in supporting health workers and in the programmes and policies that promote health. The pandemic has shown us how health is fundamental to human security. We must plan ahead and use the momentum generated by the pandemic to address health concerns and invest in prevention strategies that can reduce the burden of non-communicable diseases in particular, a key strategy being strong tobacco control.

\section{'Corona-washing'}

In 2020, the tobacco industry sought to take advantage of the COVID-19 situation ${ }^{2,3}$, misleading policymakers and the general population with so-called corporate social responsibility initiatives to whitewash its image, by providing, for instance, respiratory machines during the COVID-19 pandemic. This is particularly ironic as tobacco consumption, a risk factor for cancer, is also associated with an increased risk of developing severe COVID-19 infection ${ }^{4}$. As a public health organization, UICC $^{5}$ aims to raise awareness of every product that increases the risk of developing cancer.

Furthermore, we see that the tobacco industry is claiming that their new products (e-cigarettes and heated tobacco products) are safe by using scientific rhetoric combined with the image of innovation and technology ${ }^{6}$. This industry uses the language of the tobacco control community to sell their so-called smoke-free alternatives. As a health community, we need to expose these tactics which undermine public health and work with decision makers to ensure that

\section{AFFILIATION}

1 Union for International Cancer

Control, Geneva, Switzerland

\section{CORRESPONDENCE TO}

Yannick Romero. Union for International Cancer Control, Geneva, Switzerland.

E-mail: romero@uicc.org

\section{KEYWORDS}

World Cancer Day, prevention, interference, awareness

Received: 20 January 2021

Accepted: 25 January 2021 
countries develop and enforce strong tobacco control measures in line with the Framework Convention on Tobacco Control (FCTC).

\section{Prevention, awareness and accountability in tobacco control}

More than 8 million people die every year because of tobacco consumption, 3 million of these from cancer ${ }^{7}$, and we must continue our efforts to prevent those cancers by limiting the industry's ability to sell tobacco and disseminate misinformation about their novel products. However, the detrimental effects of tobacco are difficult to apprehend particularly for young people since cancer is a disease with late onset, meaning the signs and symptoms of cancer predominantly manifest later in life. Thus, smokers often cannot comprehend the fact that half of them will die from cancer, and the other half will have other series of health issues, such as COPD (chronic obstructive pulmonary diseases), cardio-vascular diseases and multiple comorbidities associated with smoking. In addition, it is not just smokers who are at risk, as secondhand smoke kills 1.2 million people every year.

In recent years, the global environmental movement, made famous by Greta Thunberg's \#FridaysForFuture, has involved and committed young adults around the world to address one of the globe's most pressing problems. This engagement is needed in tobacco control. A young generation can rise to build awareness about the misinformation and the tactics used by the tobacco and e-cigarette industry to maintain and expand its client base, thus exposing millions of people to addiction and poor health.

The health implications of tobacco consumption place a tremendous burden on health and health systems, but the industry always offers dubious arguments about the rights of people to use their products freely. The industry argues that if their business is restricted, then millions of jobs and revenue will be lost. However, these arguments are part of the industry playbook ${ }^{8}$, which has been developed with the collaboration of PR and marketing agencies to facilitate the dissemination of false or misleading information on novel and emerging tobacco products.

What we depict here is an entire chain that must be dismantled, and where every stakeholder must be aware that tobacco products are not only harmful to consumers, but harmful to other people who are exposed to them, and that there are serious human rights and environmental concerns related to the tobacco industry.

Preventing the use of tobacco by the next generation is crucial. Countries, governments, UN agencies, NGOs and citizens must support and act towards the implementation of efficacious and cost-effective measures to curb and stop the use of tobacco products. This can be done through taxation, ending marketing campaigns targeting teenagers and young adults, expanding smoke-free areas, and preventing the industry from lobbying governments and the public health sector, especially in low- and middle-income countries (LMICs).

We hope that 2021 will be the time when smokers refuse to be the clients of an industry which makes children work, where their products pollute oceans and endangering the tobacco farmers ${ }^{9}$ or harms economies ${ }^{10}$. Our goal is for smokers to quit and not switch to other tobacco products. We also urge policy makers and government representatives who will convene during the WHO FCTC Conference of the Parties in the Hague in November 2021 to take action on increasing taxation, on regulating tobacco products including emerging novel products or ban all activities related to the commercial marketing from the tobacco industry and the associated funded groups, including the foundation for a smoke-free world.

World Cancer Day, celebrated today, is the occasion to tell cancer advocates that tobacco is not only a health issue, but also generates important social and financial costs, it is a widespread global issue that we have to address now.

\section{REFERENCES}

1. World Cancer Day website. https://www.worldcancerday. org/. Accessed January 20, 2021.

2. Romero Y. 'Corona-washing': How the tobacco industry is advancing its interests in the midst of the pandemic. https://www.uicc.org/blog/\%E2\%80\%98coronawashing\%E2\%80\%99-how-tobacco-industry-advancingits-interests-midst-pandemic. Published April 17, 2020. Accessed January 20, 2021.

3. Unfairtobacco. False Friends: tobacco industry during Corona crisis. https://unfairtobacco.org/en/false-friendstobacco-industry-during-corona-crisis/\#/. Accessed January 20,2021. 
4. COVID-19 and smoking: resources, research and news. Tobacco Control Blog. https://blogs.bmj.com/ tc/2020/03/26/covid-19-and-smoking-resourcesresearch-and-news/. Published March 26, 2020. Accessed January 20, 2021.

5. UICC Global Cancer Control. Tobacco control: Tobacco burden. https://www.uicc.org/what-we-do/thematicareas-work/tobacco-control. Updated December 3, 2020. Accessed January 20, 2021.

6. University of Bath. Influencing Science: Commissioning Research and Reviews. Tobacco Tactics. https:// tobaccotactics.org/wiki/influencing-science-commissioningresearch-and-reviews/. Updated February 5, 2020. Accessed January 20, 2021.

7. GBD 2015 Risk Factors Collaborators. Global, regional, and national comparative risk assessment of 79 behavioural, environmental and occupational, and metabolic risks or clusters of risks, 1990-2015: a systematic analysis for the Global Burden of Disease Study 2015. Lancet. 2016;388(10053):1659-1724. doi:10.1016/S0140-6736(16)31679-8

8. World Health Organization. Tobacco Control Playbook. https://www.euro.who.int/en/health-topics/diseaseprevention/tobacco/policy/tobacco-control-playbook. Accessed January 20, 2021.

9. Unfairtobacco website. https://unfairtobacco.org/en/\#/. Accessed January 20, 2021.

10. World Health Organization. Tobacco control economics. https://www.who.int/tobacco/economics/background/ en/\#: :text=Tobacco $\% 20$ use $\% 20$ is $\% 20$ one $\% 20$ of,and $\% 20$ lost $\% 20$ productivity\%20each\%20year. Accessed January $20,2021$.

CONFLICTS OF INTEREST

The author has completed and submitted the ICMJE Form for Disclosure of Potential Conflicts of Interest and none was reported.

FUNDING

There was no source of funding for this research.

PROVENANCE AND PEER REVIEW

Commissioned; internally peer reviewed. 